\title{
Electronic model of a dubois fuzzy integration neuron
}

\author{
J.L. Pérez S.*, A. Garcés M., F. Cabiedes C., A. Miranda V. \\ Centro de Ciencias Aplicadas y Desarrollo Tecnológico \\ Universidad Nacional Autónoma de México \\ *pepito@aleph.cinstrum.unam.mx
}

\begin{abstract}
In this work, we present a fuzzy electronic neuron that has a Dubois fuzzy integration method, an activation function with a fuzzy threshold, and a fuzzy response. We generated a fuzzy sum of the input signals and a shooting threshold value defined by means of a triangular or sinusoidal membership function. We present the electronic circuits, the oscilograms of the neuron responses, the value of the fuzzy integral, and we compare their behavior with those of a conventional leaky integrator neuron.
\end{abstract}

\section{RESUMEN}

En este trabajo presentamos una neurona electrónica borrosa que contiene un integrador tipo Dubois, una función de activación con umbral borroso y respuesta borrosa. Generamos un sumador borroso de señales de entrada y un valor de disparo de umbral definido por medio de una función de membresía triangular o sinusoidal. Presentamos los circuitos electrónicos, los oscilogramas de la respuesta de la neurona, el valor de la integral borrosa, y comparamos estas características con una neurona integradora con fugas convencional.

Keywords: Biological based neuron models, artificial neuron models, Fuzzy neuron models.

\section{Introduction}

Since the first publication on neurons by Mc Cullock and Pitts in 1943 [1], many authors have tried to create physical models for neurons. The work of Pozin in 1970 [2], Deuth in 1967 [3] and Mitchell in 1963 [4] stand out among them. In the first work of Horman in 1965 [5], the neuronal model was capable of simulating only some of the properties of the biological neurons such as input signals sum, threshold, delay period, some models of neurons include some of the biological neurons adaptive properties e.g. Rosemblatt in 1960 [6], Caianiello in 1961 [7], and Widrow in 1962 [8].

Instead of using a conductance model as used by Lewis in 1977 [9], we employ a membrane potential model similar to that employed by French in 1970 [10] and Michell in 1981 [11]. The advantages of this model are simplicity in the design, simplification of the input-output relationships, facilities to establish an electronic analog model as interconnecting networks. The classical neuron model showed some of the properties of a biological neuron, such as the capacity of adding inputs, it has a variable threshold, a refractory absolute period, a relative refractory period, excitatory and inhibitory outputs, and it can have transitory response states.

As our understanding of the actual processes in the brain increases, our models will become more and more detailed. A serious problem with this kind of development is that the computer models are becoming more and more computationally intensive and memory demanding. Current models threaten to take the simulation of these models beyond the actual range of the most powerful digital computers.

An alternative leads to using analogue electronic circuits to model those neural structures; this way enables to create small, but imprecise building 
blocks [12], corresponding to, for example, individual neurons. These neurons can then be replicated many times and put on a single chip. The imprecision of the building blocks is compensated by the large number of units used and it is, therefore, not an issue in neural architectures. One advantage of conserving the architecture of neural electronic implementations is that all processing happens in parallel and in continuous time. Since there is no timemultiplexing in order to simulate multiple neurons, the circuits can function in real time. One chip models only a small number of neurons, but multiple chips can be used in parallel, so there is no obvious limit to the number of neurons in an analogue VLSI model.

There are two main reasons for building hardware models of neural models. First, a faithful neural model will allow the study of neural processing without the need of living neural tissue and without the need of simultaneously probing hundreds or thousands of neurons in the brain. Once we have neuron models with the same transfer function as biological neurons, and knowledge of their input and output connections, we can build a hardware model that allows us to study the collective behaviour of such a neural network and the individual behaviour of single neurons in the network. Finally, we can expect that neuron models might not need to replicate the biological neuron as faithful as in the first case. Once a functional model is built it will allow us to refine the amount of detail needed for a certain function and thus to create a simpler model. This is the current tendency as we can see in the following papers [13-24].

\section{Circuit description}

The neuronal model is composed of two systems: a synapses system and an axon system. The synapses system contains two circuits: analog synapses and a synapses delay circuit. The synapses analog circuit is made up of an input adder circuit, an integration circuit with variable integration time. The axon system contains the following circuits: a variable threshold circuit, an axon transmission delay circuit and a spikes output circuit. The delay circuit is made up of an oscillator, a shift registers circuit, a variable frequency astable circuit.

The classical input adder circuit was constructed using two operational amplifiers as input adders, one positive and the other negative. The output from these two adders is added in another positive circuit, which adds a membrane variable potential.

The variable threshold circuit was based on a voltage comparator circuit, with one of the voltages as threshold. The axon transmission delay circuit was constructed with a delay circuit designed from a variable time monostable. The signals blast circuit is built using an oscillator, a gate commanded by the excitatory signals. The neuron circuit achieves response transitory states as a function of the input threshold. This is achieved rising the threshold level in order to avoid the response of the circuit.

Another of the possibilities for the neuronal circuit is the frequency response change depending of the input pulse duration. This is achieved by connecting an astable circuit to the threshold, in a way that controls the time in which it is applied to the input adder. This circuit allows us to simulate the response during maintained stimuli or a maximum response and if the threshold voltage is surpassed in the adaptation circuits, it presents only a train of pulses. By controlling the threshold level, it is possible to generate an asynchronous train of response pulse. This train can be controlled by the waveform and the frequency of the input signals. In Figure (1), we show the block diagram of this classical neuron. 


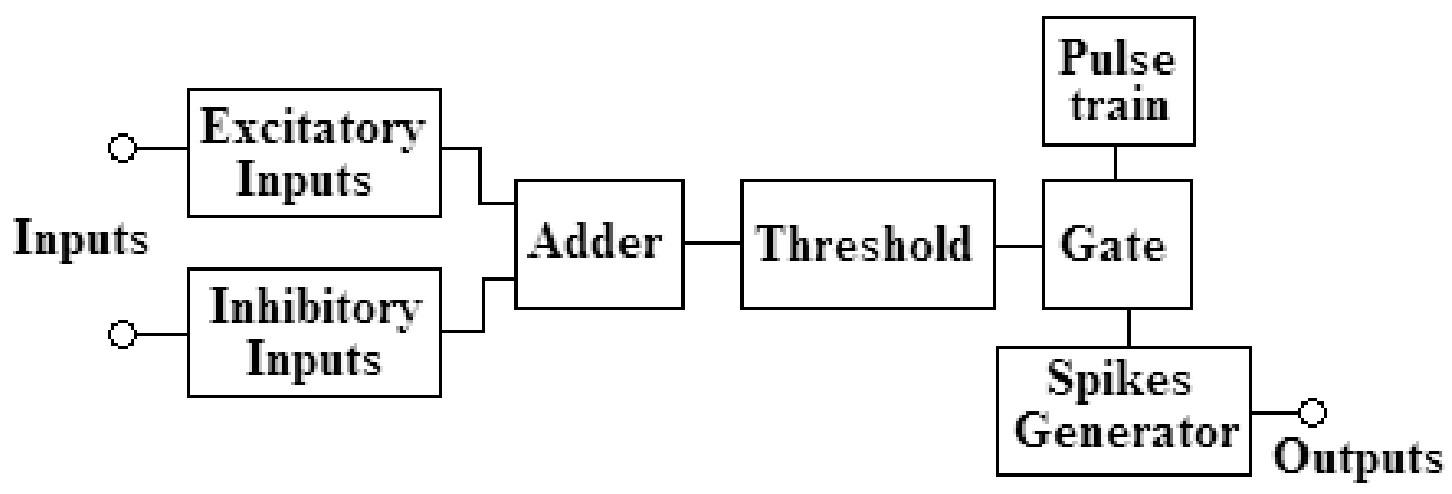

Figure 1. Block diagram of the classical neuron.

In the last five decades, since the publication of the original work of McCulloch and Pitts, there has been great interest in the neuronal networks development. The McCoulloch-Pitts model helps us to understand the characteristics of neurons as systems. It reveals the fact that the behavior of a simple nervous cell occurs not only in uncertain behavior, but also in transitions in the occurrence or absence of an event.

It is possible to consider that a model of the nervous cell behavior that satisfies these previous considerations could be the fuzzy neuron, as suggested by the generalizations of MccullochPitts [1] and Pérez-Calva's [25 and 26] models. The concept of a fuzzy neuron employs some concepts and techniques of a fuzzy sets theory, published by Zadeh [27] and applied to robots theory by Wee and Ugh [28].

Introducing the fuzzy idea within a neuron model allows electronic simulations adapted to systems that are imprecise by definition, or that have an enormous grade of complexity. Many biological systems belong to this category.
In the classical neurons, each neuron is defined by a transfer function that changes their inputs in an output. This function can be or not lineal, but if it is lineal with form $f(x)=$ ax the output is the same input modified by factor a. Input signals $x_{i}$ and weight $\omega_{\mathrm{ij}}$ are real numbers. The input signals modified by their weight are added and could be transferred by means of several output techniques like step, ramp or sigmoidal, but they could also integrate this sum before applying the response function, resulting in the typical integrator neuron.

C. Lee was first to introduce fuzzy sets in neural networks [29] and proposed a Mc Cullock and Pitts model generalization employing an intermissions value between zero and one. In 1990, Keller and Hunt [30] proposed adding a membership function to the perceptron. Yamakawa [31] introduced the idea of substituting the simple weight for a real numbers weight fuzzy set. Delgado [32] thinks that neurons have fuzzy thresholds. 


\section{A fuzzy neuron model.}

For our model, we will start from the fundamental assumption that the neuron is a kind of fuzzy process, and that it should satisfy, therefore, the following requirements; a neuron is fuzzy, according to Kandel [33], if the following conditions are satisfied:

$$
e_{j}(k) i_{j}(k)<1
$$

Where $e_{j}(k)$ is the j'th excitatory input at time $k$, and $i_{j}(k)$ is the $j$ 'th inhibitory input at time $k$. The threshold of the neuron is a real number.

The shooting neuron rules are the following:

1. All the inhibitory inputs should be zero, and

2. The sum of excitatory inputs should be greater than or equal to the neuron threshold $(\mathrm{T})$.

$$
\sum_{\mathrm{i}} \mathrm{e}_{\mathrm{i}}^{(\mathrm{k})}+\sum_{\mathrm{i}} \mathrm{i}_{\mathrm{i}}^{(\mathrm{k})} \geq \mathrm{T}
$$

3. When rules (1) and (2) are satisfied at time $t=$ $k$, the neuron is discharged at time $t=k+1$, in any another situation the neuron will remain in state of rest.
4. Neuron output $T_{i}$ is equal to zero if not discharged and any value $\omega_{\mathrm{i}}$ where: $0<\mu_{\mathrm{i}} \leq 1$ if is discharged.

So we can define a fuzzy neuron as one that has fuzzy weight and thresholds and drives fuzzy signals, fact that in the literature is recognized by the FNN3 term. In _Figure (2), a fuzzy neuron schematic model, as previously expounded, is presented.

In this work, an electronics neuron that extends the fuzzy neuron definition, generating an input signals fuzzy sum and having shooting threshold value defined by means of triangular membership functions is presented.

The input fuzzy adder is definite for our fuzzy logical equation:

$\operatorname{Min}\{1-\min (a, b), \max (a, b)\}$

Where $a=e_{j}(k)$ y $b=i_{j}(k)$, given that in fuzzy logic the tautology $P \wedge \neg P$ is valid.

In Figure (3), the proposed fuzzy neuron block diagram is shown:

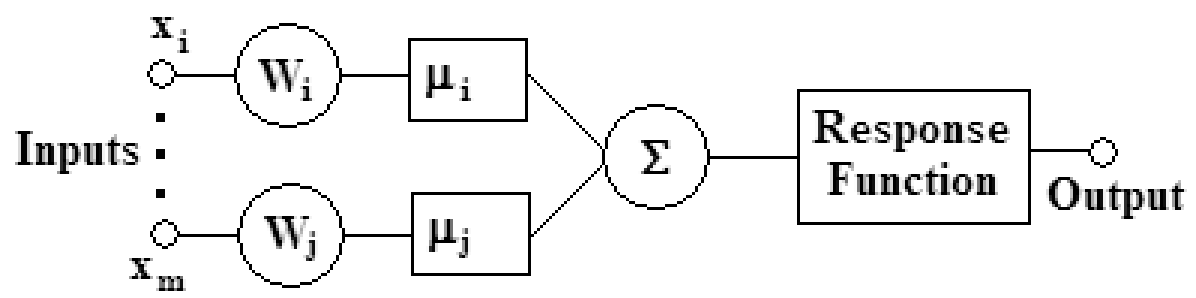

Figure 2. A fuzzy neuron blocks diagram. 


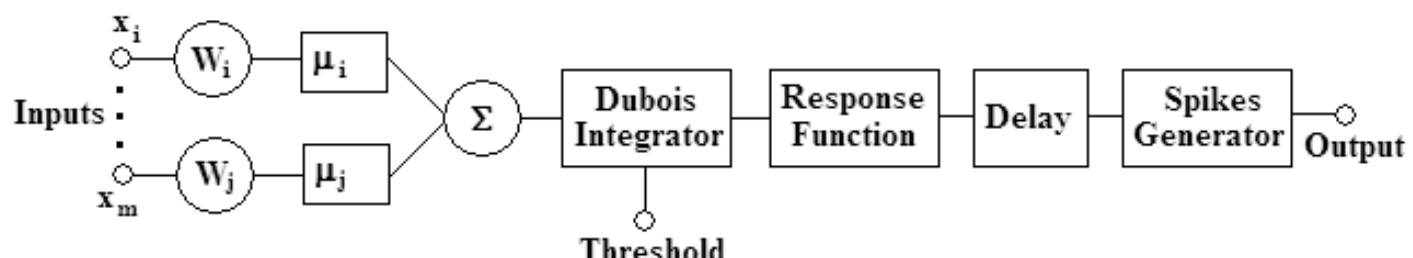

Figure 3. Proposed fuzzy neuron block diagram.

Also, the threshold condition is modified to be a time dependent function and not a fixed value, or the possibility of being biased by other neurons fuzzy values both in amplitude and frequency.

Due to the Zadeh's extension principle, it is possible to perform arithmetic operations on real fuzzy sets. In this sense, finite sums of fuzzy numbers are feasible and easy to perform, and then the problem of infinite sums (integration) of fuzzy functions may be considered. Such integration can be used to evaluate, by means of fuzzy numbers and functions, the surface of an area delimited by an ill-defined borderline. In this sense, it is possible to think of a neuron model that includes in his processes the fuzzy integration of the input signals.

We present an electronic neuron that has a Dubois fuzzy integration method [34], a fuzzy activation function with a fuzzy threshold, and a fuzzy response. The electronic design is conformed by the following circuits: the input adder circuit implementing a sum of membership functions; next, the max-min operators circuit which generates the fuzzy planes. The outputs of these operators are applied to a parallel array of electronic voltage comparators with variable thresholds. These circuits cut the fuzzy planes. The outputs of these circuits are added and then integrated to obtain the fuzzy integral.

Dubois defines the fuzzy integral as

$\mu_{\int, f(v)}=\sup _{g \in \wp} \inf _{u \in I} \mu_{f(u)}[g(u)]$, where, $v=\int_{I} g$

If we apply the Zadeh's extension principle, we can obtain

$\mu_{\int, \mathrm{f}(\mathrm{v})}=\sup _{\mathrm{g} \in \wp} \mu_{\mathrm{F}}[\mathrm{g}(\mathrm{u})]$, with $\int_{\mathrm{I}} \mathrm{g}=\mathrm{v}$.

The activation function of the fuzzy neuron is

$\mu_{\int, f(v)}=\left\{\begin{array}{cc}\sup _{g \in \wp} \mu_{g}[g(u)] & \text { si } \int_{\mathrm{I}} \mathrm{g} \geq \mathrm{h} \\ \min _{\mathrm{g} \in \wp} \mu \mathrm{g}[\mathrm{g}(\mathrm{u})] & \text { si } \int_{\mathrm{I}}^{\mathrm{g}} \mathrm{g}<\mathrm{h}\end{array}\right\}$

The block diagram of the fuzzy electronic model is shown in Figure (4). The fuzzy integrator circuit is shown in Figure (5). 


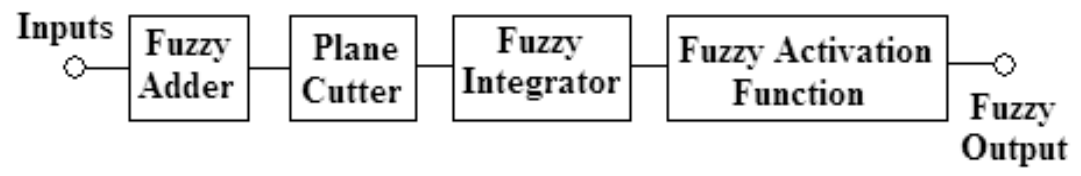

Figure 4. Block diagram of the fussy electronic neuron

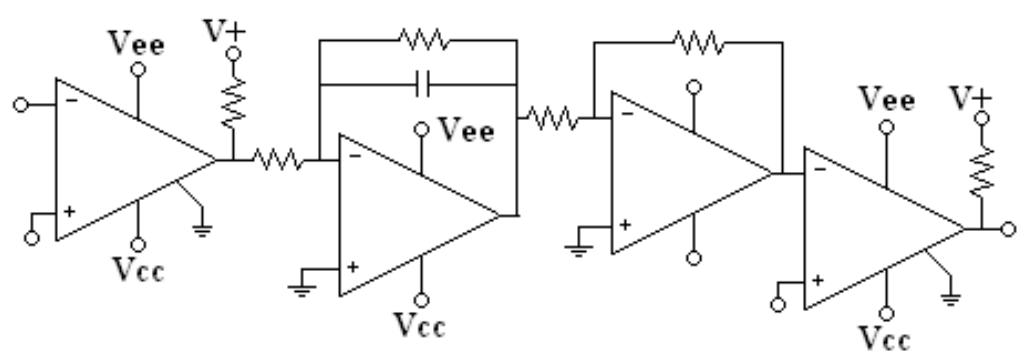

Figure 5.Fuzzy integrator circuit

The fuzziness of the neuron could be included in several levels:

1. We can say that the response is variable in accordance with the membership function that will electronically be simulated by the management of the threshold response as a function of the neuron excitement. Instead, to do that, the fuzziness resides in the neuron response, it is included by managing the threshold variation of the neuron to be excited.

2. One could also include the neuron fuzziness in the delay circuit of the axon transmission, producing delays in a fuzzy form, by membership functions in the oscillator.
3. One could also simulate fuzziness in the response of pulse train, by commanding the train via a membership function that controls an output gate.

As the simulation possibilities permit us to simulate the fuzzy response, using the neuron response coupled to a synapses function, we can establish fuzziness both on the delay time and on the shape of the transitory states response.

The neuronal model is composed of three fundamental systems: synapses, axon and fuzziness generator systems.

In Figure (6), the Neuron blocks diagram is presented.

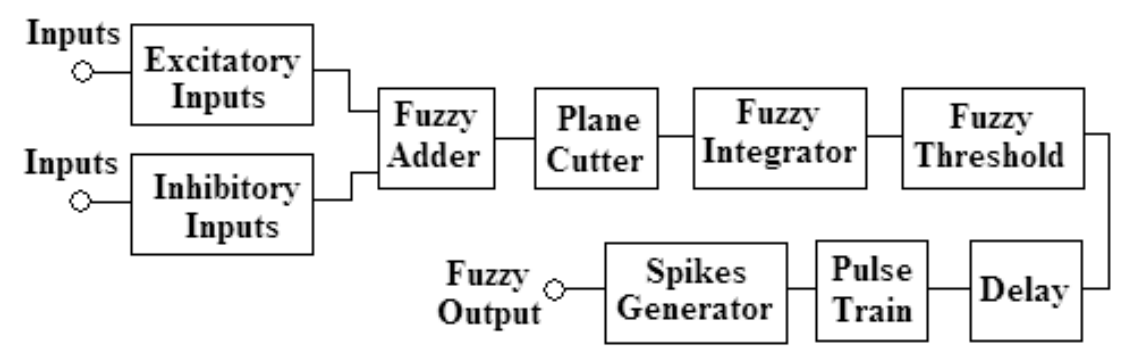

Figure 6. Fuzzy neuron blocks diagram 
The axon system is composed of the following circuits: an input fuzzy adder circuit, a Dubois fuzzy integral circuit, an axon transmission delay circuit, a signal frequency change circuit, an output circuit.

The input adder circuit is designed with two fuzzy adder circuits, one of positive inputs (excitatory), and the other of negative inputs (inhibitory). The output from these two adders is added in another fuzzy adder circuit. In Figure (7), the fuzzy adder schematic diagram is shown. Voltage is generated by a certain function in such a way that the applied voltages vary according to this function. The output from these two adders is added to another fuzzy adder circuit.

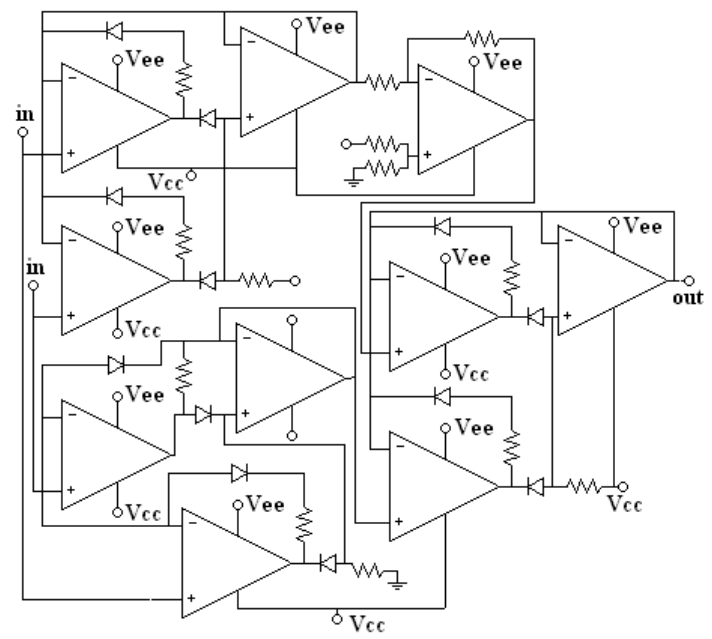

Figure 7. Schematic diagram of the fuzzy adder.

The axon transmission delay circuit is based on an astable circuit of variable time that is driven by a voltage controlled oscillator or by a signal generator. If we generate monostable control stochastic pattern, it is possible to generate fuzziness in the axon transmission speed. This allows the speeds in temporal logic neural network to have the possibility of indefinite couplings, which generates varied impulses responses.
In Figure (8), we show the delay circuit schematic diagram.

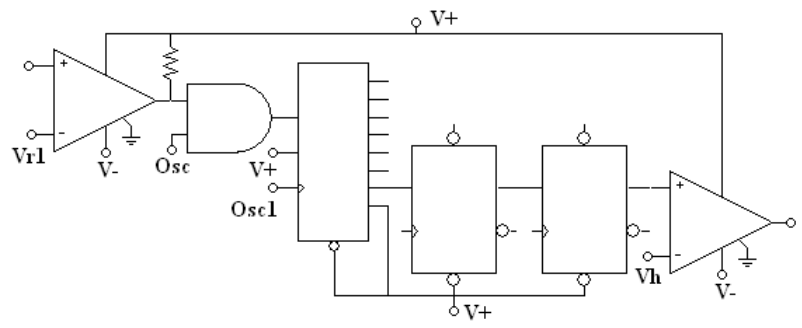

Figure 8. Circuit for an axon transmission.

We can generate the fuzziness in the generator of pulses train circuit by means of a triangular membership fuzzy pulses generator that commands the oscillator system gate. This permits us to generate asynchronous responses. In this case, the fuzziness of the system is even more difficult to drive, but it does allow the simulation of asynchronous responses. In Figure (9), we show the train pulse generator and spikes circuit schematic diagram.

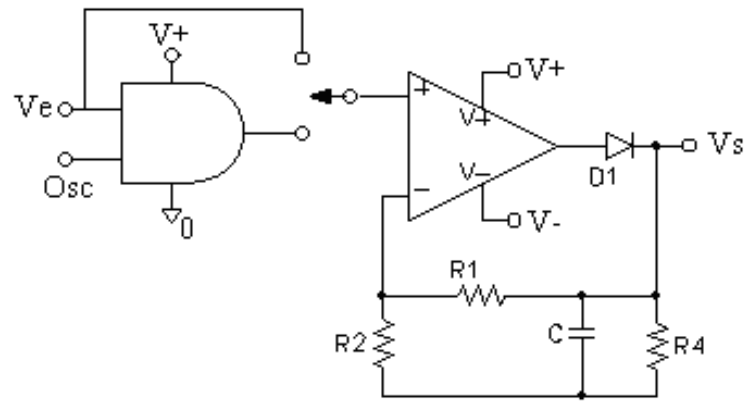

Figure 9. Spikes generator

\section{Results}

In this work we compare, with the same input signals, the classical leaky integrator neuron responses against the fuzzy neuron proposed. Two sinusoidal input signals, with the same amplitude and frequency, one for the inhibitory and the other for the excitatory inputs, were provided. 
The threshold fuzziness was driven by means of a triangular signal, and they got the following results. The classical leaky integrator neuron response graphs and the integrator phase plane will be shown. In Figure (10), the response graph of a classical leaky integrator neuron is shown.

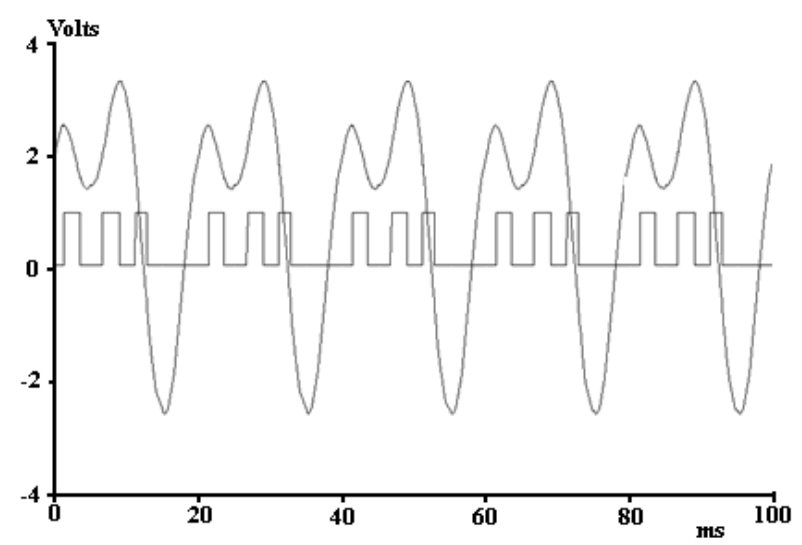

Figure 10. Classical Leaky integrator neuron response with two sinusoidal and two triangular inputs

For the same input signals and threshold conditions, the fuzzy neuron response is shown in Figure (11).

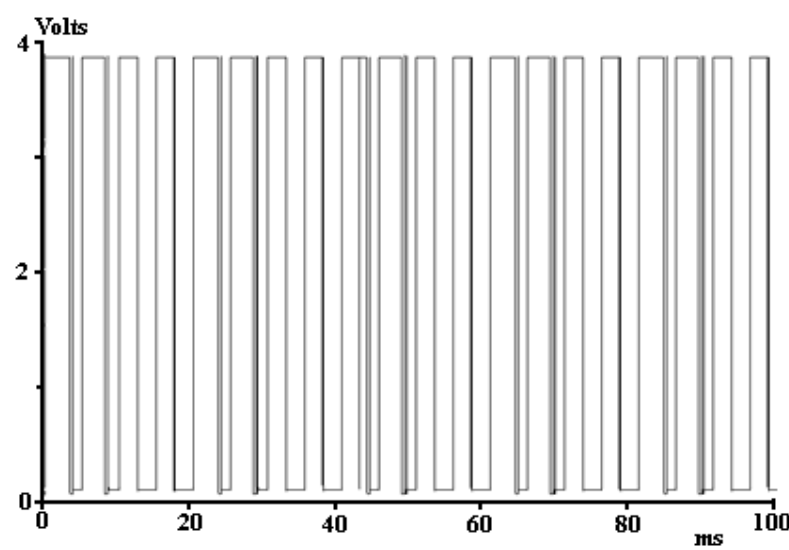

Figure 11. The fuzzy neuron response for the same input signals and threshold conditions
In Figure (12), the response of the fuzzy leaky integrator neuron with the same inputs, but with ten times the frequency, is shown:

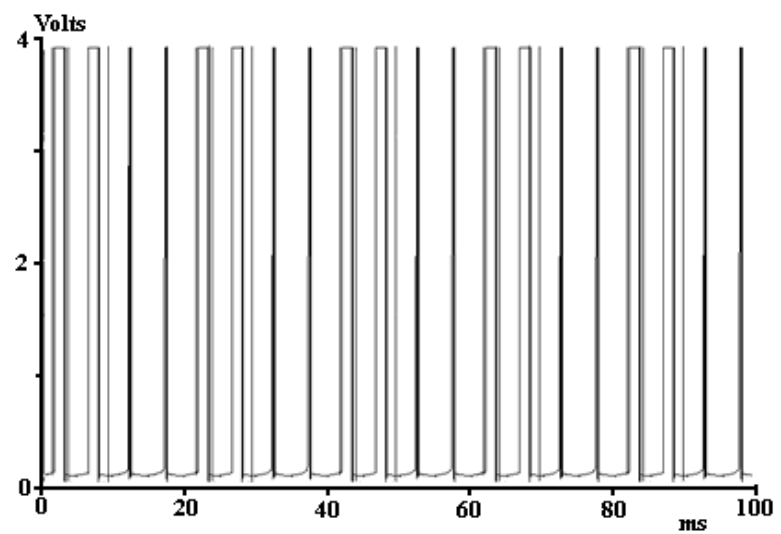

Figure 12. Response of the classical leaky integrator neuron with two square inputs, one excitatory and the other one inhibitory with triangular threshold signal.

In Figure (13), the fuzzy neuron with two square inputs, one excitatory and the other one inhibitory, with triangular threshold signal is shown:

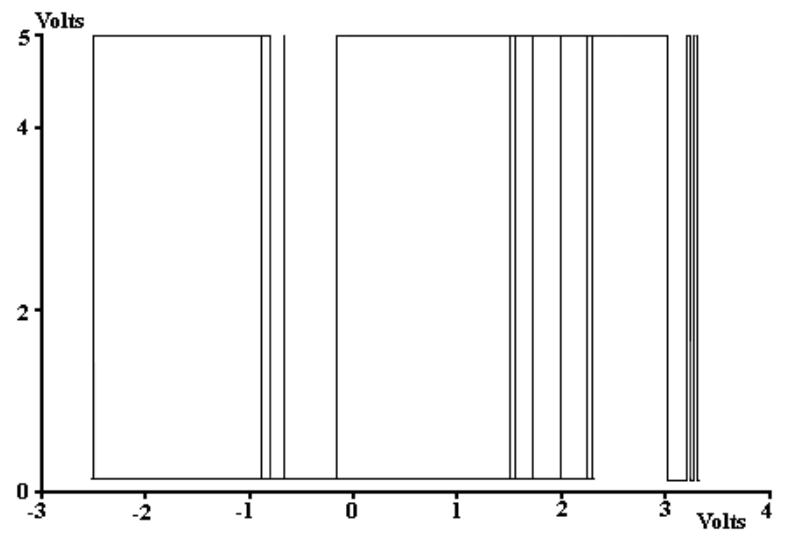

Figure 13. Fuzzy neuron input output relation.

In Figure (14), we show an operator response. 


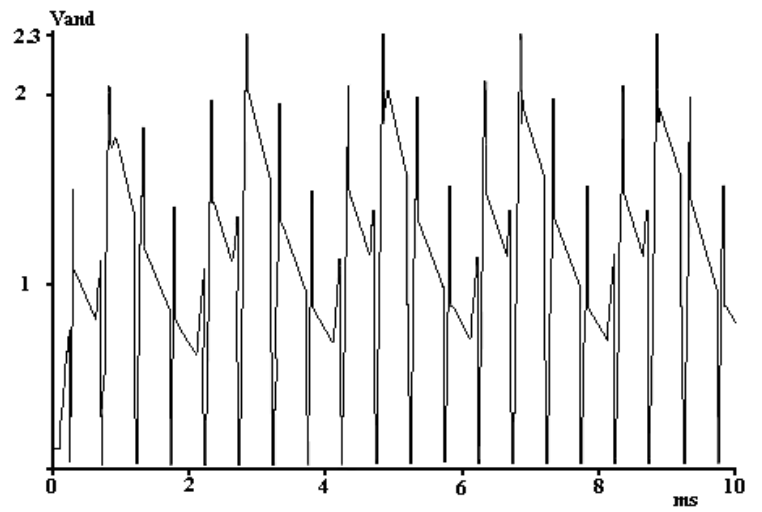

Figure 14. An operator response of the fuzzy neuron in the same conditions of input signals.

In Figure (15), we show the schematic diagram of the complete fuzzy neuron.

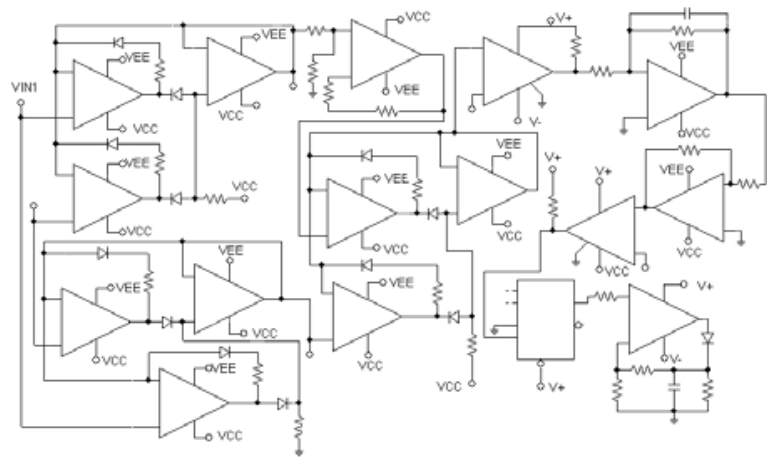

Figure 15. Schematic diagram of the fuzzy neuron

\section{References}

[1] Mc Culloch W.S., Pitts W.A. "A logical calculus of the ideas imminent in nervous activity". Bull. Math. Biophys., 5 (1943).

[2] Pozin N.V. Lyubinskii, I A, Neuron Model, Report Number FTD-HT-66-103 9 September 1966, 5 pages

[3] J.A. Deutsch, An electrophysiological stimulator with digital logic, J. exp. analysis Behav. 9 (1966), pp. 399400
[4] Mitchell RA et al.. J Appl Physiol 18, 523-533. (1963).

[5] Hormann, "Gaku: An Artificial Student," Behavioral Science, Vol. 10, No. 1 (January, 1965), pp. 88-107.

[6] Frank Rosenblatt. The Perceptron: A Probabilistic Model for Information Storage and Organization in the Brain. Psychological Review, Vol. 65, No. 6, pp. 386408, November, (1958).

[7] Caianiello, E.R. Outline of a theory of thoughtprocesses and thinking machines J. Theoret. Biol. 1, 204-235 (1961).

[8] B. Widrow and M.E. Hoff, Associative Storage and Retrieval of Digital Information in Networks of Adaptive Neurons, Biological Prototypes and Synthetic Systems, 1:160, (1962).

[9] Lewis E.R. Structural correlates of function in the anuran amphibian papilla. Scanning Electron Microsc 2. pag 429-436, (1977).

[10] French A.S., Stein R.B. "Flexible Neural Analog using integrated circuits". IEEE Trans. Biomed. Eng., 17, 248-253 (1970).

[11] Mitchell, P. Molecule, group and electron translocation through natural membranes, Biochem Soc. Symp, 22 pag 142-168, (1981).

[12] Vittoz, E.A. "Analog VLSI Signal Processing: Why, Where and How?" Journal of VLSI Signal Processing, 8 \& Analog Integrated Circuits and Signal Processing, 2744, (1994).

[13] Alexandre R.S. Romariz, KelvinWagner Optoelectronic Implementation of a FitzHugh-Nagumo Neural Model. IEEE Journal of Solid-State Circuits, 26 (7): 956-965, (1991).

[14] R. D. Pinto, P. Varona, A. R. Volkovskii, A. Szucs, H. D. Abarbanel, and M. I. Rabinovich, Synchronous behavior of two coupled electronic neurons, Phys Rev E., vol. 62, pp. 2644-56, (2000). 
[15] Y. J. Lee, Lee, J., Kim, Y.B., Ayers J., Volkovskii, A. , Selverston, A., Abarbanel, H., Rabinovich, M., "Low Power Real Time Electronic Neuron VLSI Design Using Subthreshold Techniques, IEEE Circuits and Systems, vol. 4, pp. 744-747, (2004).

[16] Shin'Ichiro Kanoh, Makoto Imai, Nozomu Hoshimiy. Analog LSI neuron model inspired by biological excitable membrane. Systems and Computers in Japan, Volume 36 Issue 6, Pages 84 - 91. (2005).

[17] A.van Schaik, Building blocks for electronic spiking neural networks. Volume 14, Issues 6-7, , Pages 6176289 (2001).

[18] Szlavik Robert B. ; Bhuiyan Abuhanif K. ; Carver Anthony ; Jenkins Frank, Neural-electronic inhibition simulated with a neuron model implemented in SPICE, IEEE Engineering in Medicine and Biology. vol. 14, n 1, pp. 109-115, (2006).

[19] A Volkovskii, S Brugioni, R Levi, M Rabinovich, A Selverston and H D I Abarbanel. Analog electronic model of the lobster pyloric central pattern generator. Journal of Physics: Conference Series 23 47-57 (2005).

20) Wu J, DiCecco J, Sun $Y$, Hill R. An analog neuron emulator for education and testing of neurophysiological instruments. Society for Neuroscience 34th Annual Meeting, San Diego, CA, October 23-27, (2004).

[21] Breau F, Maio-Cannon S, Davis R, Wu J, DiCecco J, Sun Y. The neuron emulator: an undergraduate biomedical engineering design project. 31 Northeast Bioengineering Conference, Hoboken, NJ, April 2-3, (2005).

[22] Allen I Selverston, Mikhail I Rabinovich, Henry D Abarbanel, Robert Elson, Attila Szucs, Reynaldo D Pinto, Ramon Huerta, Pablo Varona. Reliable circuits from irregular neurons: A dynamical approach to understanding central pattern generators. Journal of Physiology-Paris, Vol. 94, No. 5-6., pp. 357-374 (2000).

[23] Maeda Yoshinobu Sato, Shunsuke Makino Hideo, Bifurcation Structure of an Electronic Circuit Model for Neuronal Firings. Transactions of the Institute of
Electrical Engineers of Japan. C. ISSN:0385-4221, Vol.121-C; No.7; 1153-1159 (2001)

[24] Shinya Suenaga, Yoshihiro Hayakawa and Koji Nakajima. Design of a Neural Network Chip for the Burst ID Model with Ability of Burst Firing, IEICE Transactions on Fundamentals of Electronics, Communications and Computer Sciences E90-A(4):715723 (2007)

[25] Perez S. J.L., Calva G.: Modelo electrónico de un sistema Neuronal. Memorias del IV Simposio Nacional de Instrumentación. México. 1987.

[26] Perez S. J.L.: Diseño de un modelo de Neurona analógico. Reporte técnico. Centro de Instrumentos UNAM. (1974). Mexico. 10 pag

[27] Zadeh L.A.: Fuzzy sets. Inform Control. (1965), 338353.

[28] Wee W.C., Fu K.S. "A formulation of Fuzzy Automata and its applications as a model of Learning system". IEEE Trans. Syst. Sci. Cybernetics, (1969), SSC5.

[29] Lee., E.T. Lee. Fuzzy sets and neural networks. J. Cybernetics, (1974), 4, 83-103.

[30] Keller., D. Hunt. Incorporating fuzzy membership functions into the perceptron algorithm. IEEE Trans. Pattern Anal. Machine Intelligence. (1985), 7, 693-699.

[31] Yamakawa y S. Tomoda. A fuzzy neuron and its application to pattern recognition. Proc. Third IFSA Congr., Seattle, August 6-11, 30-38, 1989

[32] Requema y M. Delgado. "A model of fuzzy neuron". Proc. 2nd. Int. Conf. Fuzzy Logic Neural Networks. (IIZUKA'90), lizuka, Japón, July 20-24, 13-26 (1990).

[33] Kandel A. Fuzzy Switching and automata. Crane, Russak \& Company. New York. (1979).

[34] Dubois D. Towars Fuzzy Differential Calculus. Fuzzy Sets and Systems., 8, 1-17 (1982). 